\title{
Moderate Altitude Is Not Associated with Pulmonary Arterial Hypertension in Adult Patients Referred for Transcatheter Device Closure of Atrial Septal Defects
}

\author{
Jeremy Nicolarsen, $\mathrm{MD}^{1,2^{*}}$, Brett Oestreich, $\mathrm{MD}^{1}$, Sonali S. Patel, MD², Jamil Aboulhosn, $\mathrm{MD}^{3}$, \\ Wayne J. Franklin, $\mathrm{MD}^{4}$, Joseph Kay, $\mathrm{MD}^{1,2}$ \\ 1 Department of Medicine, University of Colorado Hospital, Aurora, Colorado, USA \\ 2 Department of Pediatrics, Children's Hospital Colorado, Aurora, Colorado, USA \\ ${ }^{3}$ Department of Medicine, Ronald Reagan UCLA Medical Center, Los Angeles, California, USA \\ ${ }^{4}$ Departments of Medicine and Pediatrics, Baylor College of Medicine/Texas Children's Hospital, Houston, Texas, USA
}

\begin{abstract}
Background: Pulmonary arterial hypertension (PAH) occurs in $6-10 \%$ of adults with atrial septal defects (ASDs). Although larger defect size and older age are two risk factors for the development of PAH in these patients, little is known about the impact of elevation. Thus, we aimed to determine whether the incidence of PAH is higher among patients living at moderate altitude who are referred for transcatheter device closure of an ASD. Methods: Our multicenter, retrospective cohort study included patients at least 18 years of age who were referred for device closure of an ASD (> $4 \mathrm{~mm}$ ) during a 10-year period (2003-2013). Catheterization records from three centers were reviewed. Results: Thirty-seven moderate altitude (4983-5633 ft) and 126 low altitude (74-839 ft) patients were identified $(p<0.0001)$. After controlling for age, living at moderate altitude was associated with a greater likelihood of pulmonary hypertension (odds ratio 2.29, 95\% confidence interval 1.01-5.19, $p=0.046$ ), but there was no such association with PAH ( $p$ $=0.9)$. Conclusion: Patients with ASD living at moderate altitude are more likely to exhibit pulmonary hypertension but not PAH compared with patients living at sea level. Therefore, while moderate altitude can increase pulmonary pressures, it did not contribute to the development of PAH in our cohort of patients with ASDs.

Copyright @ 2017 Science International Corp.
\end{abstract}

Fax +1 2037853346

E-Mail: jshd@scienceinternational.org

http://structuralheartdisease.org/

(c) 2017 Journal of Structural Heart Disease Published by Science International Corp. ISSN 2326-4004

Accessible online at:

http://structuralheartdisease.org/
Key Words:

Atrial septal defect - Pulmonary hypertension • Pulmonary arterial hypertension - Altitude

\section{Introduction}

Atrial septal defects (ASDs) represent $6-10 \%$ of all congenital heart defects [1]. Left unrepaired or if diagnosed later in life, ASDs are associated with pulmonary hypertension $(\mathrm{PH}$, elevated pulmonary artery pressures) or pulmonary arterial hypertension (PAH, elevated pressures and pulmonary vascular disease with normal left heart pressures). The latter condition results from years of left-to-right shunting and excessive pulmonary blood flow, which in turn can lead to irreversible pulmonary vascular remodeling characterized by medial hypertrophy, intimal thickening, and arteriolar muscularization [2]. Although ASDs cause a minority of cases of PAH associated with congenital heart disease [3, 4], progressive pulmonary vascular disease and elevated resistance can result in right heart failure, reversal of the intracardiac shunt, or Eisenmenger syndrome. Other morbidities can also arise in the setting of an unrepaired ASD, such as paradoxical embolism and stroke due to transient right-

* Corresponding Author:

Jeremy Nicolarsen, MD

Providence Adult and Teen Congenital Heart Program (PATCH)

Providence Center for Congenital Heart Disease

Providence Sacred Heart Medical Center and Children's Hospital

101 West 8th Avenue, Suite 4300, Spokane, WA 99204

Tel.: +1 509474 6707; Fax: +1 509624 9186; E-Mail: jeremy.nicolarsen@providence.org 
to-left shunt across the defect. For these reasons, patients with unrepaired ASDs are often referred for cardiac catheterization to assess pulmonary vascular resistance (PVR) and shunt fraction (Qp:Qs) followed by transcatheter device closure of the defect should there be favorable anatomy and hemodynamics.

Not all patients with unrepaired ASDs develop PAH. Although older age and defect size are independent predictors of PAH in patients with ASDs $[5,6,7]$, other risk factors are unknown. One possible risk factor may be living at higher altitude. Living at higher elevations may increase a person's risk of developing $\mathrm{PH} / \mathrm{PAH}$ due to lower oxygen tension, contributing to more substantial oxygen saturation variability, pulmonary vasoconstriction, and pulmonary vascular remodeling irrespective of the presence of structural heart defects $[8,9]$. In patients with unrepaired ASDs, little is known about these effects of elevation. Specifically, how much does living at a moderately high altitude raise pulmonary artery pressures, and is this associated with pulmonary vascular remodeling? Thus, we aimed to determine whether living at moderate altitude (4500-6000 ft or 1372-1829 m) is associated with a greater likelihood of developing $\mathrm{PAH}$ among patients referred for transcatheter device closure of ASDs.

\section{Materials and Methods}

This was a multicenter, retrospective cohort study of adult patients who were referred for transcatheter device closure of an ASD during a 10-year period (2003-2013). Catheterization databases and records from three academic centers were reviewed after individual institutional review boards approved the study. Variables extracted from records were demographic information including the patient's zip code of residence, presence of other risk factors for $\mathrm{PH}$ such as lung disease or sleep apnea, use of PH/PAH medications, and catheterization measurements including mean pulmonary artery pressure (mPAp), pulmonary capillary wedge pressure (PCWp), PVR, Qp:Qs, and defect size (balloon-sized or pre-procedure size measured by echocardiography). Elevation was defined as 'moderate altitude' if the zip code elevation was $\geq 4500 \mathrm{ft}$. Otherwise, elevation was considered 'low altitude'. The primary outcome was the presence of PAH, defined as $\mathrm{mPAp} \geq 25 \mathrm{mmHg}$, PVR $>3$ Wood units $\times \mathrm{m}^{2}$, and
$P C W p \leq 15 \mathrm{mmHg}$. The secondary outcome was the presence of $\mathrm{PH}$, defined as $\mathrm{mPAp} \geq 25 \mathrm{mmHg}$ without elevated PVR and/or with PCWp $>15 \mathrm{mmHg}$.

Patients aged 18 to 99 years with an ASD or ASD physiology (i.e., partial anomalous pulmonary venous return) were included. Patients with a patent foramen ovale, ASD $<4 \mathrm{~mm}$ (in any one imaging plane), residual ASD after prior surgical or transcatheter device closure, or other types of congenital heart disease were excluded. Raw catheterization data were collected, and Qp:Qs and PVR were independently calculated for each patient (reported values were not used). In cases in which catheterization data were incomplete, such as in reports lacking pulmonary vein and/or systemic arterial saturations, a value of $95 \%$ was used. Oxygen consumption (VO2) was derived from the LaFarge equation and table, which provides an estimated VO2 based on the patient's age and heart rate.

Study data were collected and managed using REDCap electronic data capture tools hosted by the University of Colorado [10]. Data were tested for normality using Shapiro-Wilk tests. Non-normally distributed continuous data are presented as median (interquartile range), and categorical data are presented as frequency (percentage). Comparative statistics were calculated using Wilcoxon rank-sum or Chisquare tests, as appropriate. As pulmonary pressures are known to increase with age, catheterization data were further analyzed for differences between elevation categories using logistic regression controlling for age. All analyses were performed with Statistical Analysis Software (SAS Institute, version 9.4, Cary, NC).

\section{Results}

Thirty-seven moderate altitude and 126 low altitude patients were identified. Baseline patient characteristics are outlined in Table 1. Elevations of patients' zip codes of residence ranged from 74 to 7110 $\mathrm{ft}$ above sea level, with a median elevation of $246 \mathrm{ft}$ in the low-altitude group (74-839 ft) and $5334 \mathrm{ft}$ in the moderate altitude group (4983-5633 ft, $\mathrm{p}<0.0001)$.

Defect size and PCWp were similar between groups as shown in Table 2. Despite the lack of difference in Qp:Qs between groups (median Qp:Qs 1.5 vs. $1.6, p=0.07$ ), there was a trend toward higher pulmonary artery pressures in the moderate altitude group 
Table 1. Demographic data.

\begin{tabular}{lccc}
\hline & Moderate Altitude, $\mathbf{n = 3 7}$ & Low Altitude, $\mathbf{n = 1 2 6}$ & $\boldsymbol{P}$ \\
\hline Male gender & $11(30 \%)$ & $29(23 \%)$ & 0.40 \\
Age (years) & $45(35-61)$ & $36(23-55)$ & 0.01 \\
Body surface area $\left(\mathrm{m}^{2}\right)$ & $1.8(1.7-2.1)$ & $1.8(1.6-2.0)$ & 0.13 \\
Presence of lung disease & $3(8 \%)$ & $2(2 \%)$ & 0.29 \\
Presence of obstructive sleep apnea & $1(3 \%)$ & $2(2 \%)$ & 0.39 \\
Taking PH medication & $3 / 22(25 \%)^{*}$ & $13 / 22(59 \%)^{*}$ & 0.05 \\
Elevation (ft) & $5334(4983-5633)$ & $246(74-839)$ & $<0.0001$ \\
\hline
\end{tabular}

$\mathrm{PH}=$ pulmonary hypertension

Table 2. Catheterization data.

\begin{tabular}{lccc}
\hline & Moderate Altitude, $\mathbf{n = 3 7}$ & Low Altitude, $\mathbf{n}=\mathbf{1 2 6}$ & $\boldsymbol{P}$ \\
\hline Defect size $(\mathrm{mm})$ & $20(18-27)$ & $22(17-26)$ & 0.61 \\
Qp:Qs & $1.5(1.1-2.0)$ & $1.6(1.2-2.4)$ & 0.07 \\
mPAp $(\mathrm{mmHg})$ & $25(16-33)$ & $19(15-26)$ & 0.05 \\
PCWp $(\mathrm{mmHg})$ & $11(7-14)$ & $10(7-13)$ & 0.82 \\
PVR $($ Wood units) & $1.8(1.1-2.7)$ & $1.1(0.7-1.8)$ & 0.008 \\
mPAp $\geq 25 \mathrm{mmHg}$ & $20(54 \%)$ & $37(29 \%)$ & 0.006 \\
PCWp $\leq 15 \mathrm{mmHg}$ & $32(86 \%)$ & $110(87 \%)$ & 0.90 \\
PVR $>3$ Wood units $\times \mathrm{m}^{2}$ & $6(16 \%)$ & $17(13 \%)$ & 0.68 \\
PH present† & $18(50 \%)$ & $34(27 \%)$ & 0.010 \\
PAH present & $5(28 \%)$ & $10(29 \%)$ & 0.90 \\
\hline
\end{tabular}

Qp:Qs = shunt fraction; $\mathrm{mPAp}=$ mean pulmonary artery pressure; $\mathrm{PCWp}=$ pulmonary capillary wedge pressure; $\mathrm{PVR}=$ pulmonary vascular resistance; $\mathrm{PCWp}$ = pulmonary capillary wedge pressure; $\mathrm{PH}=$ pulmonary hypertension; $\mathrm{PAH}=$ pulmonary arterial hypertension

tCriteria for PH: $\mathrm{mPAp} \geq 25 \mathrm{mmHg}$ only

‡Criteria for PAH: $\mathrm{mPAp} \geq 25 \mathrm{mmHg}, \mathrm{PCWp} \leq 15 \mathrm{mmHg}$, and PVR $>3$ Wood units $\times \mathrm{m}^{2}$

* Incomplete information was available for the entire cohort

(median mPAp $25 \mathrm{mmHg}$ vs. $19 \mathrm{mmHg}, \mathrm{p}=0.05)$, and PVR was significantly higher in the moderate altitude group than in the low altitude group (median PVR 1.8 vs. 1.1 Wood units $\times \mathrm{m}^{2}, \mathrm{p}=0.008$ ).

When evaluating components of the clinical definition of PAH (i.e., mPAp $\geq 25 \mathrm{mmHg}$, PCWp $\leq 15$ $\mathrm{mmHg}$, and PVR $>3$ Wood units $\left.\times \mathrm{m}^{2}\right)$, we found a significant difference in the prevalence of PH (mPAp $\geq 25 \mathrm{mmHg}$ ) between groups, even when controlling for age (odds ratio $2.29,95 \%$ confidence interval 1.01-5.19, $\mathrm{p}=0.046$ ). However, there was no difference in the prevalence of PAH between groups $(5 / 37(28 \%)$ at moderate altitude vs. $10 / 126(29 \%)$ at low altitude, $p=0.9$ ).

Journal of Structural Heart Disease, August 2017

\section{Discussion}

Although ASDs are a relatively small contributor to the overall prevalence of PAH in congenital heart disease, significant morbidity can occur when these defects are unrepaired. Referral for transcatheter device closure remains an important part of the management of ASDs but requires an accurate assessment of Qp:Qs and PVR with right heart catheterization (RHC) before deciding on the need and safety of device closure. If PVR is significantly elevated (i.e., $>8$ Wood units $\times \mathrm{m}^{2}$ [11]) closure of the defect may result in pressure-overload of the right ventricle with subsequent chamber enlargement and failure. By evaluating $\mathrm{RHC}$ data from a large cohort of patients who were referred for tran- 
scatheter device closure of ASDs at different elevations, we sought to determine whether elevation is an independent risk factor for the development of PAH in these patients.

We evaluated individual $\mathrm{RHC}$ hemodynamic data as well as data contributing to the clinical classifications of $\mathrm{PH}$ and $\mathrm{PAH}$ (with PAH referring to pulmonary vascular disease with elevated pulmonary artery pressures and normal left heart pressures). We found that patients living at moderate altitude showed a trend toward higher pulmonary artery pressures and that more patients living at moderate altitude met the clinical definition of $\mathrm{PH}$ than those living at low altitude. We suspect this is secondary to lower oxygen tension and more hypoxic vasoconstriction, as defect size, shunt fraction, and the presence of other causes of $\mathrm{PH}$ or use of $\mathrm{PH}$ medications were similar between groups.

In looking at PVR and PAH, the presence of which is the main contributor to morbidity in these patients and thus was our choice for primary outcome, we found that although PVR was significantly higher in the moderate altitude group, it was not elevated to the clinically important threshold that defines PAH. We believe that the higher pulmonary arterial pressures seen in the moderate altitude group contribute to higher calculated PVR (as mPAp makes up one half of the numerator in the PVR calculation, (mPAp-PCWp)/Qp), but there is likely no significant difference between groups in actual pulmonary vascular disease.

Patients with unrepaired ASDs or ASD physiology and defect size $\geq 4 \mathrm{~mm}$ were chosen because these patients are most likely to have a hemodynamically significant shunt that may predispose to the development of pulmonary vascular changes and PAH. By excluding patients with a patent foramen ovale or small defects, we intended to avoid a false underestimation of PAH prevalence in patients with interatrial communications. By excluding other types of congenital heart disease, such as ventricular septal defects, aortopulmonary connections, or other causes of left-toright shunt and excessive pulmonary blood flow, we intended to prevent a false overestimation of the burden of PAH. We did not exclude patients with multiple $\mathrm{PH}$ risk factors or multifactorial $\mathrm{PH} / \mathrm{PAH}$ because it is quite common for a patient to have more than one cause of $\mathrm{PH} / \mathrm{PAH}$, such as having an ASD and obstructive sleep apnea. By looking primarily at PAH (and not $\mathrm{PH}$ ), we focused on patients with true pulmonary vascular disease rather than those with excessive pulmonary blood flow and/or increased pulmonary pressures from lung or left heart disease.

There are several limitations of this study. First, inaccurate hemodynamic analysis using false data can often be a challenge when studying cardiac catheterizations. Specifically, a catheterization lab's computer software generates results of complex calculations of multiple variables, of which the software attempts to select variables from the data it 'sees' and/or a human operator chooses. This can result in reported calculations or results that are not physiologically correct. To avoid the problem of comparing software-generated results, we reviewed the catheterization reports closely for the values that should be used in the calculations and performed the calculations independently. In some cases, assumptions were still necessary. For instance, we used pulse oximeter saturation as a surrogate for pulmonary vein and systemic arterial saturations when these data were not available or used a value of $95 \%$ as the pulmonary vein and systemic saturation when no pulse oximeter saturation was available. Furthermore, the assumed value of $95 \%$ may vary at moderate elevation compared to sea level.

Second, we made several assumptions about our patients. We assumed that the zip code of a patient's residence at the time of chart review was the same zip code and elevation at which he or she was living at the time of the catheterization. For catheterizations 7-10 years ago, however, this may not be the case. We also assumed that a patient was living at a particular elevation long enough for it to impact his or her risk of developing PAH. Unfortunately, data regarding duration of residence at that elevation prior to catheterization was not available.

Third, residence in Denver or at elevations similar to Denver's may not be high enough to reveal a difference in the development of PAH compared with residence at sea level. Previously, $8200 \mathrm{ft}(2500 \mathrm{~m})$ or higher has been described as a threshold at which significant pulmonary vascular disease can develop [12], and Denver's population is well below that mark at $5280 \mathrm{ft}(1600 \mathrm{~m})$. Although elevation-related increases in pulmonary pressures are certainly present 
at elevations below $8200 \mathrm{ft}$, the effect of Denver's elevation (i.e., the elevation at which the majority of our moderate altitude patients were living) may be less significant than expected. Still, we feel that reporting these results is important for centers at moderate elevation.

In conclusion, patients with ASDs living at moderate altitude (4500-6000 ft or 1372-1829 m) were more likely to have $\mathrm{PH}$ or a mPAp $\geq 25 \mathrm{mmHg}$ than those living at low altitude, but there was no difference between groups in Qp:Qs or clinically relevant PVR (> 3 Wood-units $\times \mathrm{m}^{2}$ ). That is, while living at moderate altitude can raise pulmonary pressures, even when controlling for age, this did not appear to contribute to the development of PAH in our cohort of patients with ASDs.

\section{References}

1. Fyler DC. Atrial septal defect secundum. In: Keane JF, Lock JE, Fyler DC. Nadas' Pediatric Cardiology. Philadelphia, PA: Hanley \& Belfus; 1992, p. 513-524.

2. Pietra GG, Capron F, Stewart S, Leone $\mathrm{O}$, Humbert M, Robbins IM, et al. Pathologic assessment of vasculopathies in pulmonary hypertension. J Am Coll Cardio. 2004;43:25S-32S. DOI: 10.1016/j. jacc.2004.02.033

3. Steele PM, Fuster V, Cohen M, Ritter DG, McGoon DC. Isolated atrial septal defect with pulmonary vascular obstructive disease--long-term follow-up and prediction of outcome after surgical correction. Circulation. 1987;76:1037-1042. PMID: 3664992

4. Duffels MGJ, Engelfriet PM, Berger RMF, van Loon RL, Hoendermis E, Vriend JW, et al. Pulmonary arterial hypertension in congenital heart disease: An epidemiologic perspective from a Dutch registry. Int J Cardiol. 2007;120:198-204. DOI: 10.1016/j. ijcard.2006.09.017

5. Engelfriet $P$, Meijboom F, Boersma E, Tijssen J, Mulder B. Repaired and open atrial septal defects type II in adulthood: An epidemiological study of a large European co- hort. Int J Cardiol. 2008;126:379-385. DOI: 10.1016/j.ijcard.2007.04.044

6. Suchoń E, Tracz W, Podolec P, Sadowski J. Atrial septal defect in adults: The influence of age and haemodynamic parameters on the results of surgical repair. Kardiol Pol. 2006;64:470-478. PMID: 16752329

7. Motiwala A, Fatimi SH, Akhtar N, Perveen S, Khan MZ, Atiq M. Patients with congenital atrial septal defects: Effect of age at repair and defect size on pulmonary artery pressures prior to repair. Thorac Cardiovasc Surg. 2011;59:281-286. DOI: 10.1055/s0030-1250491

8. Maignan $M$, Rivera-Ch $M$, Privat $C$, León-Velarde F, Richalet J-P, Pham I. Pulmonary pressure and cardiac function in chronic mountain sickness patients. Chest. 2009;135:499-504. DOI: 10.1378/ chest.08-1094

9. Stenmark KR, Fagan KA, Frid MG. Hypoxia-induced pulmonary vascular remodeling: Cellular and molecular mechanisms. Circ Res. 2006;99:675-691. DOI: 10.1161/01. RES.0000243584.45145.3f

10. Harris PA, Taylor R, Thielke JP, Gonzalez $\mathrm{N}$, Conde JG. Research electronic data capture (REDCap) - A metadata-driven methodology and workflow process for providing translational research informatics support. J Biomed Inform. 2009;42:377381. DOI: 10.1016/j.jbi.2008.08.010

11. Lopes A, Tissot C, Ivy DD. Echocardiographic evaluation of pulmonary hypertension. In: Beghetti, M. Pediatric Pulmonary Hypertension. Munich: Elsevier Urban \& Fischer; 2011, p.187-207.

12. León-Velarde $F$, Maggiorini $M$, Reeves JT, Aldashev A, Asmus I, Bernardi L, et al. Consensus statement on chronic and subacute high altitude diseases. High Alt Med Biol. 2005;6:147-157. DOI: 10.1089/ ham.2005.6.147

Cite this article as: Nicolarsen J, Oestreich B, Patel SS, Aboulhosn J, Franklin WJ, Kay J. Moderate Altitude Is Not Associated with Pulmonary Arterial Hypertension in Adult Patients Referred for Transcatheter Device Closure of Atrial Septal Defects. Structural Heart Disease. 2017;3(4):102-106. DOI: https:// doi.org/10.12945/j.jshd.2017.003.17 